\title{
Motivos de pais e responsáveis para a não adesão à vacinação contra o Papilomavírus
}

\section{Humano: Revisão de Escopo}

Reasons for parents and guardians for non-adherence to Human Papilloma Virus vaccination: Scoping Review

Razones por las que los padres y tutores no se adhieren a la vacunación contra el Virus del Papiloma Humano: Revisión de Alcance

\author{
Aline Rafaela Neves Padilha \\ ORCID: https://orcid.org/0000-0001-7779-2817 \\ Universidade Federal de São João del Rei, Brasil \\ E-mail: anevespadilha @gmail.com \\ Maria Alice Aparecida Resende \\ ORCID: https://orcid.org/0000-0002-9919-8963 \\ Universidade Federal de São João del Rei, Brasil \\ E-mail: mariaaliceres@gmail.com \\ Mariana Dias dos Reis \\ ORCID: https://orcid.org/0000-0003-4126-6828 \\ Universidade Federal de São João del Rei, Brasil \\ E-mail: maribocaiuva@gmail.com \\ Valéria Conceição de Oliveira \\ ORCID: https://orcid.org/0000-0003-2606-9754 \\ Universidade Federal de São João del Rei, Brasil \\ E-mail: valeriaoliveira@ufsj.edu.br \\ Patrícia Peres de Oliveira \\ ORCID: https://orcid.org/0000-0002-3025-5034 \\ Universidade Federal de São João del Rei, Brasil, Brasil \\ E-mail: pperesoliveira@ufsj.edu.br \\ Natane Moreira de Carvalho \\ ORCID: https://orcid.org/0000-0003-1083-7640 \\ Universidade do Estado de Minas Gerais, Brasil \\ E-mail: natanecarvalho@gmail.com
}

\begin{abstract}
Resumo
A vacina contra o HPV foi introduzida no Programa Nacional de Imunização (PNI) do Brasil em 2014. Apesar dos esforços do Ministério da Saúde para aumentar as coberturas vacinais, o número de adolescentes vacinados não é satisfatório. Esse estudo teve como objetivo sintetizar as evidências científicas sobre os motivos que levam os pais e responsáveis a recusarem vacinar as crianças e os adolescentes contra o HPV. A metodologia empregada se deu por meio de uma Revisão de Escopo. Para a construção das etapas metodológicas, foram respeitados os critérios da Joanna Briggs Institute e o PRISMA-SCR. Realizou-se a pesquisa em seis bases de dados eletrônicos, no Portal de Periódicos da CAPES. Os dados extraídos foram analisados e sintetizados na forma narrativa. Um total de 8535 registros, foi recuperado 23 estudos que foram mantidos para revisão. Das publicações, tiveram abordagem quantitativa $(52,17 \%)$, qualitativa $(43,48 \%)$ e mista $(4,35 \%)$. Dos achados surgiram quatro categorias: segurança da vacina e medo dos eventos adversos; idade precoce e vacina contra doença sexualmente transmissível; influência cultural e religiosa; e desconhecimento e fake news. Este estudo permitiu investigar e aprofundar os conhecimentos acerca dos motivos que influenciam a tomada de decisão dos pais em relação à vacinação contra o HPV.
\end{abstract}

Palavras chave: Motivação; Recusa de vacinação; Vacinas contra Papillomavirus; Adolescente; Pais.

\section{Abstract}

The HPV vaccine was introduced in Brazil's National Immunization Program (PNI) in 2014. Despite efforts by the Ministry of Health to increase vaccine coverage, the number of vaccinated adolescents is not satisfactory. This study aimed to synthesize the scientific evidence on the reasons that lead parents and guardians to refuse to vaccinate children and adolescents against HPV. The methodology used was through a Scope Review. For the construction of the methodological steps, the criteria of the Joanna Briggs Institute and the PRISMA-SCR were respected. The research was carried out in six electronic databases, on the CAPES Journal Portal. The extracted data were analyzed and synthesized in narrative form. A total of 8535 records, 23 studies were retrieved and kept for review. Of the 
publications, they had a quantitative $(52.17 \%)$, qualitative $(43.48 \%)$ and mixed $(4.35 \%)$ approach. Four categories emerged from the findings: vaccine safety and fear of adverse events; early age and vaccine against sexually transmitted disease; cultural and religious influence; and ignorance and fake news. This study allowed us to investigate and deepen the knowledge about the reasons that influence parents' decision-making regarding HPV vaccination.

Keywords: Motivation; Vaccination refusal; Papillomavirus Vaccines; Adolescent; Parents.

\section{Resumen}

La vacuna contra el VPH se introdujo en el Programa Nacional de Inmunización (PNI) de Brasil en 2014. A pesar de los esfuerzos del Ministerio de Salud para aumentar la cobertura de la vacuna, el número de adolescentes vacunados no es satisfactorio. Este estudio tuvo como objetivo sintetizar la evidencia científica sobre las razones que llevan a los padres y tutores a negarse a vacunar a niños y adolescentes contra el VPH. La metodología utilizada fue a través de una Revisión de Alcance. Para la construcción de las etapas metodológicas se respetaron los criterios del Instituto Joanna Briggs y el PRISMA-SCR. La investigación se realizó en seis bases de datos electrónicas, en el Portal de Revistas CAPES. Los datos extraídos se analizaron y sintetizaron en forma narrativa. Se recuperaron y conservaron para revisión un total de 8535 registros, 23 estudios. De las publicaciones, tuvieron un enfoque cuantitativo $(52,17 \%)$, cualitativo $(43,48 \%)$ y mixto $(4,35 \%)$. De los hallazgos surgieron cuatro categorías: seguridad de la vacuna y temor a eventos adversos; edad temprana y vacuna contra enfermedades de transmisión sexual; influencia cultural y religiosa; e ignorancia y fake news. Este estudio nos permitió investigar y profundizar en el conocimiento sobre los motivos que influyen en la toma de decisiones de los padres sobre la vacunación contra el VPH.

Palabras clave: Motivación; Negativa a la vacunación; Vacunas contra Papillomavirus; Adolescente; Padres.

\section{Introdução}

O papilomavírus humano (HPV) é a doença sexualmente transmissível de maior incidência global, estima-se que existam cerca de 600 milhões de pessoas infectadas pelo HPV no mundo e que $80 \%$ da população sexualmente ativa já tenham entrado em contato com o vírus em algum momento da vida (Giraldo, 2008; WHO, 2016; Forman et al., 2013). A contaminação ocorre por meio do contato direto com epitélios da pele e da mucosa e pode chegar a $65 \%$ logo após o contato com uma pessoa infectada (Giraldo, 2008). O HPV está associado ao câncer cervical, além das verrugas genitais, as lesões précancerosas do trato anogenital masculino e feminino, bem como os cânceres de cabeça e pescoço (Giraldo, 2008; Lipold et al., 2013; McLaughlin-Drubin \& Münger,2009).

Diante desse contexto, diferentes países aderiram à política de vacinação com intuito de diminuir o número de casos de lesões pré-cancerígenas causadas pelo HPV. Para atingir esse objetivo desenvolveram-se imunizantes nas formas bivalente, quadrivalente e nonavalente, os quais protegem contra os sorotipos do vírus e impedem o surgimento de neoplasias intraepiteliais de alto grau (Santos \& Dias, 2018). Existem no mínimo 15 tipos de HPV precursores de câncer, que possuem alto risco ou possibilidade de acarretar infecções duradouras e lesões pré-cancerígenas. Dentre os HPV de elevado risco oncogênico (16,18,45 e 56), os tipos 16 e 18 estão associados a casos de câncer cervical. Os HPV 6 e 11, são causadores por condilomas genitais sendo considerados não oncogênicos. (Lopalco,2017)

A vacina bivalente protege contra HPVs dos subtipos 16 e 18, prevenindo lesões pré-cancerosas na genitália e no colo do útero feminino. A forma quadrivalente confere proteção adicional contra os tipos HPV6 e HPV11, prevenindo verrugas anogenitais em mulheres e homens, além de impedir lesões genitais pré-cancerosas de colo do útero, vulva e vagina em mulheres, e anal em ambos os sexos, estes últimos relacionados aos HPVs 16 e 18 (Araújo et al., 2013; Ministério da Saúde, 2017). A vacina nonavalente foi desenvolvida a partir da quadrivalente, e abrange proteção contra os subtipos de HPV 31, 33, 45, 52 e 58 (Lopalco, 2017).

A implementação da vacina contra o HPV ocorreu de forma gradual em cada Programa Nacional de Imunização, cada país foiresponsável pela logística e ações para a oferta da vacina, que acontecia de acordo com a epidemiologia da doença e o financiamento disponível (OMS, 2017, Moura, 2019). A Austrália foi um dos primeiros países a disponibilizar a vacina gratuitamente em 2007 (Patel et al., 2018). Na América Latina, todos os países disponibilizam a vacina quadrivalente 
Research, Society and Development, v. 11, n. 1, e28211124792, 2022

(CC BY 4.0) | ISSN 2525-3409 | DOI: http://dx.doi.org/10.33448/rsd-v11i1.24792

gratuitamente para o público alvo (Luciani et al., 2019. Já em países de renda média e baixa o ritmo de implementação foi mais lento, por exemplo, no Senegal (LaMontagne et al., 2017).

No Brasil, desde março de 2014, o Ministério da Saúde oferece gratuitamente a vacina quadrivalente contra o HPV por meio do Programa Nacional de Imunizações (PNI) (Santos \& Dias, 2018). A princípio, as vacinas foram oferecidas nas unidades básicas de saúde e escolas, com a meta de atingir $80 \%$ de cobertura vacinal do grupo alvo. Inicialmente, apenas as meninas recebiam gratuitamente a vacina. Em 2016, tendo em vista a importância epidemiológica da infecção pelo HPV no homem, os meninos também foram incluídos no calendário vacinal (Souza, 2020; Santos \& Dias, 2018).

Apesar dos esforços do Ministério da Saúde para aumentar as coberturas vacinais, o número de adolescentes vacinados não é satisfatório, a cobertura vacinal reduziu para 60\% na segunda dose (Ministério da Saúde, 2018; Lobão, 2018). Segundo a Sociedade Brasileira de Imunizações, em 2020, 70\% das meninas de 9 a 15 anos tomaram a primeira dose da vacina contra o HPV, os meninos de 11 a 14 anos tiveram um percentual de um pouco mais de 40\%. Na segunda dose, esses índices foram de aproximadamente $40 \%$ para as meninas e $30 \%$ para os meninos (SBIM, 2020).

Somado à baixa cobertura vacinal, outro fato preocupante é que mais de um quarto dos jovens de 13 a 17 anos já iniciaram a vida sexual e 39,8\% destes alegaram não ter feito uso de preservativos durante a primeira relação sexual (IBGE, 2016). Desta forma, as baixas coberturas vacinais, o início da vida sexual e a não utilização de preservativos deixa esses adolescentes mais suscetíveis a contrair o HPV (Santos, 2018).

$\mathrm{O}$ ato de negar a utilização das vacinas induz a atitudes que colocam em risco não só a saúde individual do não vacinado, mas, de todos à sua volta (Mizuta et al., 2018). Nesse contexto, identificar os principais motivos de não vacinação das crianças e adolescentes por pais e responsáveis, proporciona subsídio para que a equipe de saúde realize ações mais efetivas a fim de melhorar a cobertura vacinal contra o HPV. Dessa forma, esse estudo teve como objetivo identificar e sintetizar as evidências científicas sobre os motivos que levam os pais e responsáveis recusarem vacinar as crianças e os adolescentes contra o HPV.

\section{Metodologia}

Trata-se de uma revisão de escopo, com protocolo de pesquisa registrado no Open Science Framework (https://osf.io/7fxmk/), desenvolvido com base nas recomendações do guia internacional PRISMA-ScR (Tricco et al., 2018) e no método proposto pelo Joanna Briggs Institute, Reviewers (Peters et al., 2020). Foram seguidas as cinco etapas de revisão de escopo (Peters et al., 2020) a saber: 1) identificação da questão de pesquisa; 2) identificação dos estudos relevantes; 3) seleção dos estudos; 4) análise dos dados; e, 5) agrupamento, síntese e apresentação dos dados. A questão de pesquisa deste estudo foi elaborada seguindo a combinação mnemônica PCC (Peters et al., 2020), P: População - Responsáveis por escolares em idade vacinal contra o HPV; C: conceito - Motivos da não vacinação; C: Contexto - vacinação contra o HPV, sendo estabelecida a seguinte questão norteadora: Quais os motivos da não vacinação contra o HPV relatado por responsáveis de criança/adolescente?

Os critérios de inclusão preestabelecidos foram artigos originais, publicados na íntegra em inglês, espanhol e português, com textos completos disponíveis on-line nas bases de dados selecionadas que versavam sobre os motivos da não vacinação contra o HPV. O limite temporal foram artigos publicados a partir de 2014. Esse período é justificado pela inclusão da vacina contra o HPV no âmbito do SUS no Brasil. Houve exclusão dos estudos que não contemplaram a pergunta norteadora, editoriais, relatos de experiência, ensaios teóricos, estudos de revisão, estudo de caso único e estudos duplicados.

As bases de dados utilizadas foram a National Library of Medicine (PubMed), Cumulative Index to Nursing and Allied Health Literature (CINAHL), Embase, Web of Science, Scopus, Literatura Latino-Americana e do Caribe em Ciências da 
Saúde (LILACS). A busca em tais bases de dados foi realizada de março a abril de 2021, de forma concomitante em todas elas, por três revisoras. Em todas essas bases, a pesquisa aconteceu por meio da busca avançada. Inicialmente, realizou-se uma pesquisa nas bases de dados PubMed e CINAHL, com o objetivo de identificar os descritores mais utilizados nos estudos que abordavam a temática de interesse. Após, foram avaliadas as publicações para identificar os descritores relacionados aos itens da estratégia de pesquisa.

Selecionaram-se os seguintes descritores controlados de terminologia preconizada pelo Medical Subject Headings (MeSH) e/ou os Descritores em Ciências da Saúde (DeCS): Motivação / Motivation / Motivación / AND Vacinas contra Papillomavirus / Papillomavirus Vaccines / Vacunas contra Papillomavirus AND Pais / Parents / Padres OR Mães / Mothers / Madres OR Pai / Fathers / Padre OR Avós / Grandparents / Abuelos AND Adolescente / Adolescent / Adolescente OR Criança / Child / Niño AND Recusa de Vacinação / Vaccination Refusal / Negativa a la Vacunación. Os termos foram buscados em sua equivalência em inglês, espanhol e português. As buscas realizadas nas bases citadas acima ocorrerammediante a utilização do Portal de Periódicos da CAPES, por meio da Comunidade Acadêmica Federada (CAFe), com seleção de acesso proveniente da instituição de ensino superior Universidade Federal de São João del-Rei (UFSJ). O controle de busca utilizado nessas bases, a localização, a estratégia de busca e a utilização de filtros para refinar a coleta, quando possível, estão descritos no Quadro 1.

Quadro 1 - Descritores, operadores boleanos e filtros utilizados nas buscas. Divinópolis, Minas Gerais, Brasil, 2021.

\begin{tabular}{|c|c|c|}
\hline Base de dados & Estratégia de busca e filtros utilizados & Total \\
\hline $\begin{array}{l}\text { MEDLINE via PubMed } \\
\text { (https://pubmed-ncbi-nlm- } \\
\text { nih.ez32.periodicos.capes.gov.br/) }\end{array}$ & $\begin{array}{l}\text { Motivation AND Papillomavirus Vaccines AND Parents OR } \\
\text { Mothers OR Fathers OR Grandparents AND Adolescent OR } \\
\text { Children OR Child AND Vaccination Refusal } \\
\text { Filtros utilizados: Texto completo; Ano de publicação: } 2014-2021\end{array}$ & 500 artigos \\
\hline $\begin{array}{l}\text { LILACS } \\
\text { (http://lilacs.bvsalud.org/http://pesquisa.bvsalud.org/ } \\
\text { portal/advanced/) }\end{array}$ & $\begin{array}{l}\text { Motivation AND Papillomavirus Vaccines AND Parents OR } \\
\text { Mothers OR Fathers OR Grandparents AND Adolescent } \\
\text { Children OR Child AND Vaccination Refusal }\end{array}$ & 0 artigos \\
\hline $\begin{array}{l}\text { Scopus } \\
\text { (https://www- } \\
\text { scopus.ez32.periodicos.capes.gov.br/search/form.uri? } \\
\text { display=basic\#basic) }\end{array}$ & $\begin{array}{l}\text { Motivation AND Papillomavirus Vaccines AND Parents OR } \\
\text { Mothers OR Fathers OR Grandparents AND Adolescent } \\
\text { Children OR Child AND Vaccination Refusal } \\
\text { Filtros utilizados: Acesso aberto; Ano de publicação: } 2018\end{array}$ & 1 artigos \\
\hline $\begin{array}{l}\text { Web of Science } \\
\text { (https://www- } \\
\text { webofscience.ez32.periodicos.capes.gov.br/wos/wosc } \\
\text { c/basic-search) }\end{array}$ & $\begin{array}{l}\text { Motivation AND Papillomavirus Vaccines AND Parents OR } \\
\text { Mothers OR Fathers OR Grandparents AND Adolescent OR } \\
\text { Children OR Child AND Vaccination Refusal } \\
\text { Filtros utilizados: Ano: } 2014 \text { - 2021; Acesso Aberto; Categoria: } \\
\text { Enfermagem; Tipo de documento: Artigo }\end{array}$ & 2985 artigos \\
\hline $\begin{array}{l}\text { CINAHL } \\
\text { (http://web-a- } \\
\text { ebscohost.ez32.periodicos.capes.gov.br/ehost/search/ } \\
\text { basic?vid=0\&sid=3cef7bc7-9003-4a47-9de4- } \\
\text { 877b49848a44\%40sdc-v-sessmgr03) }\end{array}$ & $\begin{array}{l}\text { Motivation AND Papillomavirus Vaccines AND Parents OR } \\
\text { Mothers OR Fathers OR Grandparents AND Adolescent OR } \\
\text { Children OR Child AND Vaccination Refusal } \\
\text { Filtros utilizados: Texto completo; Data de publicação: } 2014-2021\end{array}$ & 5047 artigos \\
\hline $\begin{array}{l}\text { Embase } \\
\text { (https://www- } \\
\text { embase.ez32.periodicos.capes.gov.br/?phase=continu } \\
\text { eToApp\#search) }\end{array}$ & $\begin{array}{l}\text { Motivation AND Papillomavirus Vaccines AND Parents OR } \\
\text { Mothers OR Fathers OR Grandparents AND Adolescent } \\
\text { Children OR Child AND Vaccination Refusal } \\
\text { Filtros utilizados: Origem embase; Doença: Papilomavírus infecção; } \\
\text { Tipo de documento: Artigo; Ano de publicação: } 2014-2021\end{array}$ & 2 artigos \\
\hline
\end{tabular}

Fonte: Elaborado pelos autores (2021).

Os estudos encontrados nas bases de dados, por meio das chaves de busca, foram exportados para o Programa Rayyan QCRI. Posteriormente, foi realizada a remoção dos estudos duplicados e a realização da leitura dos títulos e resumos por três revisores utilizando o método triplo cego a fim de identificar estudos potencialmente elegíveis de acordo com os critérios 
Research, Society and Development, v. 11, n. 1, e28211124792, 2022

(CC BY 4.0) | ISSN 2525-3409 | DOI: http://dx.doi.org/10.33448/rsd-v11i1.24792

estabelecidos. A inclusão ou exclusão dos artigos em que houve dúvidas e/ou divergências entre os revisores quanto à sua elegibilidade foram resolvidas por consenso entre os mesmos.

Para a extração dos resultados, que consistia em separar, sumarizar e relatar os elementos essenciais encontrados em cada pesquisa foi utilizado um instrumento estruturado, o qual favoreceu a síntese, interpretação dos dados, natureza e distribuição dos estudos incorporados na revisão (Melnyk \& Fineout-Overholt, 2011). Para a compilação e comunicação dos resultados, um quadro com as principais características dos estudos como título, autores e ano de publicação, país, abordagem metodológica, periódico ou instituição foi elaborado, visando apresentar uma visão geral de todo o material. Além de descrição numérica dos resultados, uma descrição temática foi organizada de acordo com a natureza dos estudos.

Segundo a resolução 466/2012, não foi necessária a apreciação do comitê de ética por se tratar de um estudo de natureza bibliográfica, contudo, os aspectos éticos e os direitos autorais foram respeitados e referenciados. (Brasil, 2012)

\section{Resultados}

As estratégias de busca permitiram recuperar 8535 estudos, sendo 111 artigos duplicados, restando 8424 estudos. A leitura de títulos e resumos resultou na manutenção de 33 artigos, os quais tinham como fenômeno de interesse os motivos da não vacinação contra o HPV.

Destes, a partir da leitura do texto integral, foram excluídos 10 artigos porque tratavam da opinião dos profissionais e apresentavam motivos relacionados à logística da vacinação. A Figura 1 exibe o fluxograma do processo de seleção das publicações desta revisão com base na recomendação Prisma (Tricco et al., 2018). 
Research, Society and Development, v. 11, n. 1, e28211124792, 2022

(CC BY 4.0) | ISSN 2525-3409 | DOI: http://dx.doi.org/10.33448/rsd-v11i1.24792

Figura 1 - Processo de busca, de exclusão e de seleção dos estudos encontrados.

\section{Identificação de estudos por meio de banco de dados}

\begin{tabular}{|c|c|c|}
\hline 氖 & $\begin{array}{l}\text { Artigos identificados: } \\
\text { Databases }(\mathrm{n}=8535) \\
\text { Medline/PubMed - } 500 \\
\text { Embase - } 2 \\
\text { Scopus - } 1\end{array}$ & $\begin{array}{l}\text { Registros removidos antes da triagem } \\
\text { Registros duplicados removidos }\end{array}$ \\
\hline : & $\begin{array}{c}\downarrow \\
\text { Registros selecionados } \\
(\mathrm{n}=8424) \\
\end{array}$ & $\begin{array}{l}\text { Registros excluídos } \\
\qquad(\mathrm{n}=8391) \\
\text { Excluídos por não apresentarem elementos que }\end{array}$ \\
\hline & $\begin{array}{l}\text { Registros selecionados } \\
\qquad(\mathrm{n}=33)\end{array}$ & Registros excluídos: $(\mathrm{n}=10)$ \\
\hline 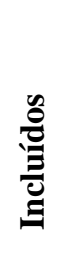 & $\begin{array}{l}\text { Registros selecionados } \\
\qquad(\mathrm{n}=23)\end{array}$ & $\begin{array}{l}\text { Abordar a opinião dos profissionais não } \\
\text { abrangendo a opinião dos responsáveis pelas } \\
\text { crianças/adolescentes: } 4 \text {; } \\
\text { Apresentar motivos relacionados a logística da } \\
\text { vacinação: } 3 \text {; }\end{array}$ \\
\hline
\end{tabular}

Fonte: Fluxo do processo de seleção dos estudos da Scoping Review, adaptado do PRISMA-ScR (Tricco et al., 2018). Divinópolis, Minas Gerais, Brasil, 2021

Os resultados foram apresentados com uma descrição das características dos estudos e, na sequência apresentam-se as quatro categorias evidenciadas a partir dos estudos selecionados: segurança da vacina e medo dos eventos adversos; idade precoce e vacina contra doença sexualmente transmissível; influência cultural e religiosa; o desconhecimento e as fake news.

O Quadro 2 apresenta as principais características dos estudos. O maior número de publicações $(\mathrm{n}=5)$ foi em 2019 (Hirth et al., 2019; Gyan et al., 2019; Sanchez et al., 2019; Thomas et al., 2019; Tatar et al., 2019) seguido por (n=4) em 2017 (Gilkey et al., 2017; Albright et al., 2017; Foster et al., 2017; Grandahl et al., 2017). Quanto à procedência editorial, os estudos foram publicados em 15 periódicos científicos, sendo o maior número de estudos encontrados no Human Vaccines \& 
Immunotherapeutics, com 5 estudos (Gilkey et al., 2017; Gilbert et al., 2016; Krawczyk et al., 2015; Thomas et al., 2019; Tatar et al., 2019), seguido pelo periódico Vaccine em que foram encontrados 3 artigos (Hirth et al., 2019; Waller et al., 2020; Szilagyi et al., 2020).

Quanto à nacionalidade dos artigos, doze (52,17\%) foram desenvolvidos nos Estados Unidos seguido por Canadá em que foram selecionados três $(13,05 \%)$ artigos, Suécia e Reino Unido dois $(8,69 \%)$ artigos e os demais países com um $(4,35 \%)$ estudo. No que se refere à abordagem metodológica, doze publicações utilizaram a abordagem quantitativa (52,17\%) e dez a qualitativa (43,48\%), enquanto um estudo utilizou ambas as abordagens (4,35\%) como apresentado Quadro 2.

Quadro 2: Caracterização dos artigos segundo título, autor, ano de publicação, país de realização do estudo, abordagem metodológica, periódico, Divinópolis, Minas Gerais, Brasil, 2021.

\begin{tabular}{|c|c|c|c|c|}
\hline Título & Autores e Ano & País & $\begin{array}{l}\text { Abordagem } \\
\text { metodológica }\end{array}$ & Periódico ou Instituição \\
\hline $\begin{array}{l}\text { 1-Parents who refuse or delay HPV vaccine: } \\
\text { Differences in vaccination behavior, beliefs, } \\
\text { and clinical communication preferences }\end{array}$ & (Gilkey et al., 2017) & EUA & Estudo quantitativo & $\begin{array}{l}\text { Human Vaccines \& } \\
\text { Immunotherapeutics }\end{array}$ \\
\hline $\begin{array}{l}\text { 2-Acceptability of human papillomavirus } \\
\text { vaccine: parent survey }\end{array}$ & (Baddouh et al., 2018) & Marrocos & Estudo quantitativo & BioMed Research International \\
\hline $\begin{array}{l}\text { 3-Estimates and determinants of HPV non- } \\
\text { vaccination and vaccine refusal in girls } 12 \text { to } \\
14 \text { y of age in Canada: Results from the } \\
\text { Childhood National Immunization Coverage } \\
\text { Survey, } 2013\end{array}$ & (Gilbert et al., 2016) & Canadá & Estudo quantitativo & $\begin{array}{l}\text { Human Vaccines \& } \\
\text { Immunotherapeutics }\end{array}$ \\
\hline $\begin{array}{l}\text { 4-Parents' decision-making about the human } \\
\text { papillomavirus vaccine for their daughters: } \\
\text { II. Qualitative results }\end{array}$ & $\begin{array}{l}\text { (Krawczyk et } \\
\text { al.,2015) }\end{array}$ & Canadá & Estudo qualitativo & $\begin{array}{l}\text { Human Vaccines \& } \\
\text { Immunotherapeutics. }\end{array}$ \\
\hline $\begin{array}{l}\text { 5-Salient factors among hispanic parentes in } \\
\text { South Florida rural communities for } \\
\text { vaccinating their children against human } \\
\text { papillomavirus }\end{array}$ & (Pineda, 2020) & EUA & Estudo qualitativo & Journal of Pediatric Nursing \\
\hline $\begin{array}{l}\text { 6-Variations in reason for intention not to } \\
\text { vaccinate across time, region, and by } \\
\text { race/ethnicity, NIS-Teen (2008-2016) }\end{array}$ & (Hirth et al., 2019) & EUA & Estudo quantitativo & Vaccine \\
\hline $\begin{array}{l}\text { 7-Not the right time: why parents refuse to } \\
\text { let their daughters have the human } \\
\text { papillomavirus vaccination }\end{array}$ & (Grandhal et al., 2014) & Suécia & Estudo qualitativo & $\begin{array}{l}\text { Acta Pediatrica } \\
\text { Nurturing the child }\end{array}$ \\
\hline $\begin{array}{l}\text { 8-Parenta Refusal of Human papillomavirus } \\
\text { vaccine multisite stdudy }\end{array}$ & (Kinder, 2018) & EUA & $\begin{array}{l}\text { Estudo de métodos } \\
\text { mistos }\end{array}$ & Journal of Pediatric Health Care \\
\hline $\begin{array}{l}\text { 9-Factors Associated with HPV Vaccination } \\
\text { among Cambodian American Teenagers }\end{array}$ & (Lee et al., 2016) & EUA & Estudo quantitativo & Public Health Nursing \\
\hline $\begin{array}{l}\text { 10-HPV vaccine acceptance among African- } \\
\text { American mothers and their daughters: an } \\
\text { inquiry grounded in culture }\end{array}$ & (Gyan et al., 2019) & EUA & Estudo qualitativo & Ethnicity \& Health \\
\hline $\begin{array}{l}\text { 11-Reasons for Lack of HPV Vaccine } \\
\text { Initiation in NIS-Teen Over Time: Shifting } \\
\text { the focus from gender and sexuality to } \\
\text { Necessity and safety }\end{array}$ & (Beavis et al., 2018) & EUA & Estudo quantitativo & Journal of Adolescent Health \\
\hline $\begin{array}{l}\text { 12-Perception about barriers and facilitators } \\
\text { of the school-based HPV vaccine program of } \\
\text { Manizales, Colombia: A qualitative study in } \\
\text { school-enrolled girls and their parents }\end{array}$ & (Sanchez et al., 2019) & Colômbia & Estudo qualitativo & Preventive Medicine Reports \\
\hline
\end{tabular}


Research, Society and Development, v. 11, n. 1, e28211124792, 2022

(CC BY 4.0) | ISSN 2525-3409 | DOI: http://dx.doi.org/10.33448/rsd-v11i1.24792

\begin{tabular}{|c|c|c|c|c|}
\hline $\begin{array}{l}\text { 13-Perceptions of and barriers to vaccinating } \\
\text { daughters against Human Papillomavirus } \\
\text { (HPV) among mothers in Hong Kong }\end{array}$ & (Siu, 2014) & China & Estudo qualitativo & BMC Women's Health \\
\hline $\begin{array}{l}\text { 14-Decision-making about HPV vaccination } \\
\text { in parents of boys and girls: A population- } \\
\text { based survey in England and Wales }\end{array}$ & (Waller et al., 2020) & Reino Unido & Estudo quantitativo & Vaccine \\
\hline $\begin{array}{l}\text { 15-A short report: parents HPV vaccine } \\
\text { knowledge in rural South Florida }\end{array}$ & $\begin{array}{l}\text { (Thomas, Caldera, } \\
\text { Mauer, 2019) }\end{array}$ & EUA & Estudo quantitativo & $\begin{array}{l}\text { Human Vaccines \& } \\
\text { Immunotherapeutics }\end{array}$ \\
\hline $\begin{array}{l}\text { 16-Prevalence and characteristics of HPV } \\
\text { vaccine hesitancy among parents of } \\
\text { adolescents across the US }\end{array}$ & (Szilagyi et al., 2020) & EUA & Estudo quantitativo & Vaccine \\
\hline $\begin{array}{l}\text { 17-Noninitiation and Noncompletion of } \\
\text { HPV Vaccine Among English- and Spanish- } \\
\text { Speaking Parents of Adolescent Girls: A } \\
\text { Qualitative Study }\end{array}$ & (Albright et al., 2017) & EUA & Estudo qualitativo & Academic pediatrics \\
\hline $\begin{array}{l}\text { 18-Exploring human papillomavirus } \\
\text { vaccination refusal among ethnic minorities } \\
\text { in England: A comparative qualitative study }\end{array}$ & (Foster et al., 2017) & Reino unido & Estudo qualitativo & Psychooncology \\
\hline $\begin{array}{l}\text { 19-Delay and refusal of human } \\
\text { papillomavirus vaccine for girls, national } \\
\text { immunization survey-teen, } 2010\end{array}$ & (Dorell et al., 2014) & EUA & Estudo quantitativo & Clinical Pediatrics (Phila) \\
\hline $\begin{array}{l}\text { 20-Using the precaution adoption process } \\
\text { model to clarify human papillomavirus } \\
\text { vaccine hesitancy in canadian parents of } \\
\text { girls and parents of boys }\end{array}$ & (Tatar et al., 2019) & Canadá & Estudo quantitativo & $\begin{array}{l}\text { Human Vaccines \& } \\
\text { Immunotherapeutics }\end{array}$ \\
\hline $\begin{array}{c}\text { 21-To Consent or Decline HPV Vaccination: } \\
\text { A Pilot Study at the Start of the National } \\
\text { School-Based Vaccination Program in } \\
\text { Sweden }\end{array}$ & (Grandahl et al., 2017) & Suécia & Estudo quantitativo & Journal of School Health \\
\hline $\begin{array}{l}\text { 22-I don't know if I'm making the right } \\
\text { decision': French mothers and HPV } \\
\text { vaccination in a context of controversy }\end{array}$ & (Ward et al., 2016) & França & Estudo qualitativo & Health, Risk \& Society \\
\hline $\begin{array}{l}\text { 23-Protecting our Khmer daughters: ghosts } \\
\text { of the past, uncertain futures, and the human } \\
\text { papillomavirus vaccine }\end{array}$ & (Burke et al., 2015) & EUA & Estudo qualitativo & Ethnicity \& Health \\
\hline
\end{tabular}

Fonte: Elaborado pelos autores (2021).

A análise dos textos integrais dos 23 artigos incluídos permitiu identificar os principais motivos da não vacinação contra o HPV de cada publicação, os quais foram organizados em categorias, que serão apresentadas a seguir conforme análise temática.

\subsection{Segurança da vacina e medo dos eventos adversos:}

Dos artigos analisados, 17 apresentaram como motivos para a não vacinação a desconfiança quanto à segurança do imunobiológico, o medo e as incertezas quanto aos eventos adversos, bem como as complicações à longo prazo que possam ser causados pela vacinação (Baddouh et al., 2018; Gilbert et al., 2016; Krawczyk et al., 2015; Fernandez-Pineda et al., 2020; Hirth et al., 2019, Grandahl et al., 2014; Kinder, 2018; Gyan et al., 2019; Beavis et al., 2018; Sanchez et al., 2019; Siu, 2014; Waller et al., 2020; Szilagyi et al., 2020; Albright et al., 2017; Forster et al., 2017; Dorell et al., 2014; Ward et al., 2017). Segundo os autores a introdução da vacina contra o HPV no Brasil pode ser considerada recente, se comparada a outras vacinas da infância. Isso pode gerar dúvidas sobre a segurança e eficácia da mesma, tornando-se uma das principais razões para não vacinação das crianças e adolescentes (Gilbert et al., 2016; Krawczyk et al., 2015; Hirth et al., 2019; Kinder, 2018; 
Research, Society and Development, v. 11, n. 1, e28211124792, 2022

(CC BY 4.0) | ISSN 2525-3409 | DOI: http://dx.doi.org/10.33448/rsd-v11i1.24792

Gyan et al., 2019; Beavis et al., 2018; Siu, 2014; Waller et al., 2020; Albright et al., 2017; Forster et al., 2017; Dorell et al., 2014; Ward et al., 2017)

Nos estudos analisados os pais/responsáveis desconfiam da segurança dos componentes encontrados na vacina contra o HPV e sentem-se preocupados com as reações adversas que estes poderiam causar, bem como receio de que a criança esteja sendo usada como "cobaia". Eles acreditam também, que os componentes da vacina podem ser nocivos ao organismo da criança ou adolescente, por se tratar de corpo jovem e em desenvolvimento (Foster et al., 2017; Ward et al., 2017; Grandhal et al., 2014). E outros ainda afirmam não vacinar as crianças devido a problemas médicos já existentes e o receio de que a vacina pudesse piorar essa condição (Grandhal et al., 2014). Além disso, os participantes relatam acreditar na importância da imunidade natural e citam outras formas de prevenção do câncer cervical (Ward et al., 2017).

\subsection{Idade precoce e vacina contra doença sexualmente transmissível:}

Os estudos demonstram que os pais e responsáveis têm medo da vacina estimular os adolescentes a iniciarem a vida sexual precocemente (Gilkey et al., 2017; Krawczyk et al., 2015; Fernandez-Pineda, 2020; Hirth et al., 2019; Grandahl et al., 2014; Kinder, 2018; Gyan et al., 2019; Beavis et al., 2018; Sanchez et al., 2019; Siu, 2014; Waller et al., 2020; Albright et al., 2017; Forster et al., 2017; Dorell et al., 2014; Grandahl et al., 2017). Os responsáveis julgam que não seja o momento de vacinar os adolescentes, pois estes não realizam atividades sexuais, não sendo necessária a prevenção de infecções sexualmente transmissíveis (IST). (Gilkey et al., 2017; Fernandez-Pineda, 2020; Hirth et al., 2019; Grandahl et al., 2014; Beavis et al., 2018; Siu, 2014; Waller et al., 2020; Albright et al., 2017; Dorell et al., 2014). Um estudo menciona o relato de um pai que justifica a não vacinação da filha por presumir que a adolescente não terá relações sexuais antes dos 16/17 anos e alegam que é impossível a mesma conhecer meninos durante esse período, tornando a vacinação precoce desnecessária (Krawczyk et al., 2015).

A relação sexual dos jovens é considerada, por muitos, como um tabu (Fernandez-Pineda, 2020; Hirth et al., 2019; Grandahl et al., 2014; Kinder, 2018; Sanchez et al., 2019; Albright et al., 2017). Nos estudos analisados os entrevistados afirmam que ao discutir abertamente sobre essa temática, poderia encorajar as crianças e adolescentes a iniciarem a vida sexual precocemente (Fernandez-Pineda, 2020; Grandahl et al., 2014; Kinder, 2018; Albright et al., 2017). Os achados indicam, ainda, preocupação dos pais em oferecer a vacina e gerar uma falsa sensação de segurança na prevenção das IST (Krawczyk et al., 2015; Grandahl et al., 2014; Siu, 2014; Waller et al., 2020; Albright et al., 2017, Forster et al., 2017; Dorell et al., 2014). Outro estudo aponta a crença entre os entrevistados de que a prevenção contra IST deveria ocorrer por meio de educação e não a partir de vacinas (Sanchez et al., 2019). Além disso, enfatizam que os adolescentes são muito jovens para receber o imunobiológico (Hirth et al., 2019; Kinder, 2018; Gyan et al., 2019; Siu, 2014; Waller et al., 2020; Albright et al., 2017).

\subsection{Influência cultural e religiosa:}

Outros motivos que também reduziram a aceitação da vacinação do HPV foram às crenças religiosas e a influência cultural (Pineda, 2020; Hirth et al., 2019; Grandhal et al., 2014; Gyan et al., 2019; Albright et al., 2017; Foster et al., 2017). Segundo os estudos analisados, as crenças possuem grande poder de persuasão para não aderência à imunização contra o HPV. Algumas religiões determinam a abstinência sexual antes do matrimônio, o que justifica o motivo da não vacinação, pelo argumento da iniciação da vida sexual dos filhos pós-casamento (Pineda, 2020; Hirth et al., 2019; Gyan et al., 2019; Albright et al., 2017). Os responsáveis justificam ainda que seus filhos terão apenas um parceiro, reduzindo o risco de exposição, considerando assim, a vacina desnecessária (Grandhal et al., 2014). E alguns pais ainda argumentam que o câncer é propósito divino, influenciando diretamente no ato de não vacinar (Foster et al., 2017). 
Research, Society and Development, v. 11, n. 1, e28211124792, 2022

(CC BY 4.0) | ISSN 2525-3409 | DOI: http://dx.doi.org/10.33448/rsd-v11i1.24792

Um estudo apresenta o relato de um entrevistado que aponta a influência cultural como motivo da não vacinação. Nesse relato o homem possui maior poder na tomada de decisão da casa e não aceita discutir sobre o tema relacionado à vacina do HPV com a esposa, essa, aceita a decisão do marido e não vacina a filha contra o HPV. Portanto, nos remete à influência cultural relacionada ao ato de negar a vacinação contra o HPV, devido ao assunto ser evitado, considerado um tabu a ser discutido em ambiente domiciliar (Madeline et al., 2020).

\subsection{O desconhecimento e as fake news:}

Os 23 artigos trazem o desconhecimento sobre a doença e a vacina e a disseminação de fake news como motivo para a não aderirem à mesma. $\mathrm{O}$ desconhecimento dos pais em relação à doença e sua proteção através da vacinação se sobrepõem ao conhecimento acerca da importância e dos benefícios da imunização, comprometendo a adesão vacinal contra o HPV e a proteção das crianças e adolescentes. Em diversos artigos os responsáveis entrevistados relataram que nunca ouviram o termo HPV, ou receberam pouca ou nenhuma informação sobre a doença, o que os levou a questionarem e desconfiarem da real gravidade da doença e da eficácia da vacina na proteção contra o câncer causado pelo HPV (Gilkey et al., 2017; Baddouh et al., 2016; Gilbert et al., 2016; Krawczyk et al., 2015; Pineda, 2020; Hirth et al., 2019; Grandhal et al., 2014; Kinder, 2018; Siu, 2014; Lee et al., 2016; Beavis et al., 2018; Dorell et al., 2014; Sanchez et al., 2019, Szilagyi et al., 2020; Albright et al., 2017; Thomas, Caldera, Mauer, 2019; Waller et al., 2020).

Além do desconhecimento sobre a patologia e a vacina, rumores infundamentados e circulação de notícias falsas interferem no processo decisório de vacinação dos pais levando-os a crer que o HPV poderia ser evitado por outros meios que não a vacinação (Foster et al., 2017). Muitos pais afirmam que suas principais fontes de informação sobre a vacinação contra o HPV são os meios de comunicação e a internet (Grandhal et al., 2017). Esses meios de informação estão sujeitos a abordarem fake news e informações equivocadas sobre o assunto, levando os mesmos a interpretarem de forma errônea a gravidade da enfermidade e relatarem danos associados à vacina sem que haja devida comprovação da veracidade dos fatos, prejudicando assim a vacinação (Tatar et al., 2019; Grandhal et al., 2017; Ward et al., 2016; Burke et al., 2015; Gyan et al., 2019).

\section{Discussão}

Os Estados Unidos foram os precursores da vacinação contra o HPV, talvez por isso o maior número de publicações realizadas por eles (Beavis et al., 2018). Em comparação com outras regiões, os Estados Unidos obtiveram cobertura vacinal do HPV com índices acima do esperado pelo estado na primeira dose, no entanto, na dose seguinte houve uma queda. Após a recomendação de vacinar os meninos de 13 a 17 anos em 2011, a cobertura vacinal ampliou para esta categoria a partir do ano de 2016, alcançando cobertura vacinal de 56,0\% na primeira dose e 31,5\% na segunda dose (Podgorski et al.,2019).

Os estudos demonstraram que os principais motivos para a baixa adesão à vacina contra o HPV, estão relacionados à: desconfiança dos pais ou responsáveis quanto à segurança da vacina, aos possíveis eventos adversos, além dos efeitos no organismo a curto ou longo prazo, a disseminação de informações errôneas, o desconhecimento sobre o HPV e a vacina, a religião e cultura dos responsáveis, bem como os tabus sobre temas relacionados à sexualidade. Estes achados podem estar relacionados à incorporação recente da vacina ao PNI e a disseminação de informações inadequadas sobre os eventos adversos, que levam aos pais e responsáveis a se sentirem inseguros quanto a essa forma de prevenção (Silva et al., 2020). Entretanto, o imunizante é necessário, vantajoso e seguro, uma vez que permite a proteção individual e coletiva (Silva et al., 2020). Outro achado encontrado como motivo para não aceitação dos responsáveis na vacinação dos adolescentes contra o HPV apresentase relacionado ao custo da vacina, se tratando de países como os EUA onde são necessários investimentos monetários para aplicação do imunobiológico (Hirth et al., 2019; Dorell et al., 2014). Ressalta-se que no Brasil a vacinação é oferecida 
gratuitamente por meio do Sistema Único de Saúde, contudo, encontram-se ainda baixas coberturas vacinais (Santos \& Dias, 2018; Lobão, 2018)

Os imunizantes, de uma forma geral, são comprovadamente eficazes, eles possibilitam o controle e/ou erradicação de várias doenças, contudo, é esperado que eventos adversos pós-vacinação aconteçam. Ao analisar o Sistema de Informações do Programa Nacional de Imunizações de Eventos Adversos Pós-Vacinação - SIEAPV, do Brasil, os principais eventos adversos pós-vacinação contra o HPV, notificados foram: palidez, hipotensão, desmaio e síncope e reações locais como dor, calor, eritema ou rubor e prurido local. Estes sintomas frequentemente evoluíram para cura sem sequelas e a maioria deles é associada à ansiedade e medo da imunização. Os pesquisadores concluíram então, que mesmo diante de tais ocorrências a vacinação é uma estratégia favorável e segura contra a infecção (Silva et al., 2020).

A vigilância dos eventos adversos pós-vacinação é uma atividade fundamental para garantir a segurança e eficácia das vacinas. Informações atualizadas e abrangentes são necessárias como forma de subsídio na tomada de decisão quando surgem rumores sobre eventos adversos. Os profissionais de saúde precisam estar cientes sobre seu papel diante desse processo, realizando ações educativas com pais e responsáveis com o intuito de disseminar informações acessíveis ao público, uma vez que por meio do conhecimento permite minimizar a insegurança em relação à vacina contra o HPV (Oliveira et al., 2020).

A vacina contra o HPV é efetivamente melhor no início da adolescência, entre nove e quatorze anos, antes do início da atividade sexual (Bednarczyk et al., 2019; Ministério da Saúde, 2017). Nessa fase, a resposta imune é mais eficaz se comparada a adultos jovens, diminuindo o risco de desenvolver lesões com potencial cancerígeno nos vacinados (Bednarczyk et al., 2019; Brasil, 2017). Contudo, o fato da atividade sexual dos jovens ainda ser considerada um tabu e dos pais e responsáveis terem medo de falar abertamente sobre a temática por acreditarem que incentivariam os filhos a iniciarem a vida sexual de forma precoce, podem dificultar a adesão vacinal na adolescência (Fernandez-Pineda et al., 2020; Hirth et al., 2019; Kinder, 2018; Sanchez et al., 2019; Albright et al., 2017), mesmo após os estudos apontarem a alta eficácia da vacina e proteção duradoura durante os anos (Schward et al., 2019; Villa et al., 2020).

Os trabalhos ressaltam que a religião e a cultura são fatores que exercem forte influência na tomada de decisão da população, em relação à vacinação e por isso interferem diretamente na não adesão vacinal (Pineda, 2020; Hirth et al 2019; Grandhal et al., 2014; Gyan et al., 2019; Albright et al., 2017; Foster et al., 2017), o que contribui para a redução das taxas de imunização, prevenção de doenças, retorno de doenças que haviam sido erradicadas e, consequentemente, o aumento de casos das doenças já existentes (Barbosa et al., 2020). Os responsáveis pelos adolescentes acreditam que a imunização contra o HPV não seja necessária já que a adoção de uma cultura de abstinência sexual até o casamento preveniria as doenças sexualmente transmissíveis (Pineda, 2020; Hirth et al., 2019; Gyan et al., 2019; Albright et al., 2017). Contudo, ressalta-se que os jovens têm iniciado a vida sexual cada vez mais precoce, sendo esse acontecimento de desconhecimento dos pais, deixando os adolescentes sem orientações necessárias e vulneráveis a doenças, assim como, gravidez (Lara, 2019).

$\mathrm{O}$ desconhecimento em relação à doença e a vacina foi o principal motivo apresentado pelos pais para não aderirem à vacinação (Gilkey et al., 2017; Baddouh et al., 2016; Gilbert et al., 2016; Krawczyk et al., 2015; Pineda, 2020; Hirth et al., 2019; Grandhal et al., 2014; Kinder, 2018; Lee et al., 2016;Gyan et al .,2019 Beavis et al., 2018; Sanchez et al., 2019, Siu,2014; Waller et al., 2020; Thomas, Caldera, Mauer, 2019; Szilagyi et al., 2020; Albright et al., 2017; Foster et al., 2017; Dorell et al.,2019;Tatar et al.,2019; Grandhal et al., 2017;Ward et al., 2016; Burke et al.,2015), este influencia diretamente no processo decisório de vacinar, pois pais que não possuem acesso a informações, sobre a vacina e sua eficácia, não sentem necessidade de realizá-la, diminuindo assim a taxa de cobertura vacinal.(Restivo et al., 2018).

Em relação às estratégias utilizadas para a vacinação do HPV, as parcerias com as escolas influenciam de forma significativa para o aumento da adesão vacinal, (Podgorski et al., 2019). No Brasil, a vacinação de HPV alcançou uma adesão 
Research, Society and Development, v. 11, n. 1, e28211124792, 2022

(CC BY 4.0) | ISSN 2525-3409 | DOI: http://dx.doi.org/10.33448/rsd-v11i1.24792

de $92,3 \%$ entre os anos de 2014 e 2015, quando o público-alvo eram meninas de onze a treze anos e a estratégia adotada foi à vacinação no ambiente escolar. No ano seguinte, quando foram incluídas meninas de nove a onze anos, houve um declínio da adesão. O Ministério da Saúde atribui esta queda, em grande parte, ao fato de muitas escolas terem deixado de se envolver com a vacinação (Podgorski et al., 2019). Assim como no Brasil, para aumentar a cobertura vacinal, os Estados Unidos utilizaram a imunização como pré-requisito para a frequência escolar, sob jurisdição dos estados. Essa atitude mostrou ser eficaz em aumentar a cobertura vacinal para crianças e adolescentes, o que reforça que a parceria entre o ensino e os serviços de saúde atua como uma boa estratégia a ser adotada (Podgorski et al.,2019).

Uma revisão sistemática realizada por Guzman-holst et al.,2020 mostrou que as situações que dificultam a vacinação na América Latina são fatores de acessibilidade como falta de infraestrutura, falta de conhecimento e capacitação de profissionais de saúde. A falta de informação sobre as vacinas, tanto pelos profissionais como pela população e fatores individuais, como por exemplo, a aceitação ou recusa da vacina causada pela falta de confiança e de conhecimento dos benefícios e da necessidade da vacina também são fatores dificultadores (Guzman-holst et al., 2020).

Desde a implantação da vacina, discursos errôneos, dispersos em meios de comunicação e redes sociais, vêm prejudicando as ações de saúde pública. O amplo acesso a essas redes de comunicação e a disseminação em massa dessas informações, aumentam a resistência relacionada à vacina. A divulgação dos eventos adversos pós-vacinação assume papel de destaque na mídia, e dificulta ainda mais a percepção da população acerca dos benefícios da vacinação (Guedes et al., 2017; Godoi, Melo, Silva, 2019). Dessa forma, a disponibilização de informações seguras e corretas sobre as consequências causadas pela doença e os benefícios da vacinação são estratégias fundamentais para a conscientização dos pais a respeito da vacinação. Estas devem ser repassadas conforme a capacidade de entendimento das diferentes classes sociais considerando sua escolaridade e habilidade para acessar e processar tais informações (Almeida et al., 2020).

Os profissionais de saúde devem se atualizar e ampliar seus conhecimentos sobre o tema, além disso, em meio acadêmico, pesquisas precisam ser realizadas a fim de aumentar conhecimento sobre o HPV e a vacina, pois os resultados desses estudos poderão subsidiar as informações que serão ofertadas à população melhorando a cobertura vacinal e fortalecendo o planejamento de ações educativas que também possuem esse mesmo propósito (Silva et al., 2018).

Dentre as limitações do processo da elaboração do estudo, podemos citar que pode haver pesquisas publicadas em outros idiomas que não foram incluídas, além disso, não foram considerados outros estudos que provavelmente existem em outras bases de indexação.

\section{Considerações Finais}

Este estudo permitiu investigar e aprofundar os conhecimentos acerca dos motivos que influenciam na tomada de decisão dos pais em relação à vacinação contra o HPV. Muitos rumores, mitos, medos e crenças interferem na decisão de vacinar. O entendimento dos motivos da não vacinação, expressos na perspectiva dos pais e responsáveis de adolescentes, pode subsidiar o profissional na elaboração da educação em saúde com o intuito de uma maior promoção do conhecimento para os pais, responsáveis, crianças e adolescentes, com a finalidade de compreenderem a patologia e importância da vacinação contra o HPV e, consequentemente, estes buscarem a imunização.

\section{Agradecimentos}

Este estudo permitiu investigar e aprofundar os conhecimentos acerca dos motivos que influenciam na tomada de decisão dos pais em relação à vacinação contra o HPV. Muitos rumores, mitos, medos e crenças interferem na decisão de vacinar. O entendimento dos motivos da não vacinação, expressos na perspectiva dos pais e responsáveis de adolescentes, pode 
Research, Society and Development, v. 11, n. 1, e28211124792, 2022

(CC BY 4.0) | ISSN 2525-3409 | DOI: http://dx.doi.org/10.33448/rsd-v11i1.24792

subsidiar o profissional na elaboração da educação em saúde com o intuito de uma maior promoção do conhecimento para os pais, responsáveis, crianças e adolescentes, com a finalidade de compreenderem a patologia e importância da vacinação contra o HPV e, consequentemente, estes buscarem a imunização.

\section{Referências}

Albright, K., Barnard, J., O'Leary, S. T, Lockhart, S., Jimenez-Zambrano, A., Stokley, S. \& Kempe, A. (2017). Não início e não conclusão da vacina contra o HPV entre pais de meninas adolescentes que falam inglês e espanhol: um estudo qualitativo. Pediatria acadêmica, 17 (7), $778-784$.

Almeida, R. C. A. A., Castro, J. M., Castro Oliveira, T. V., de Oliveira, T. F., Araújo, D. A., Azevedo, M. A., \& Costa, W. J. T. (2020). Cobertura vacinal ANTI-HPV e motivos de não vacinação. Revista Eletrônica Acervo Enfermagem, 2, e2600-e2600.

Araujo, S. C. F. D., Caetano, R., Braga, J. U., \& Costa \& Silva, F. V (2013). Eficácia das vacinas comercialmente disponíveis contra a infecção pelo papilomavírus em mulheres: revisão sistemática e metanálise. Cadernos de Saúde Pública, 29, 32-44.

Baddouh, N., Rada, N., Elalouani, F. E., Draiss, G., \& Bouskraoui, M. (2018). Aceitabilidade da vacina do vírus do papiloma humano: pesquisa dos pais. The Pan African Medical Journal, 31, 71-71.

Barbosa, L. F. (2020). Recusa vacinal e o impacto no ressurgimento de doenças erradicadas. Brazilian Journal of Surgery and Clinical Research

Beavis, A., Krakow, M., Levinson, K., e Rositch, AF (2018). Razões para a falta de iniciação da vacina contra o HPV em NIS-Teen ao longo do tempo: mudando o foco de gênero e sexualidade para necessidade e segurança. Journal of Adolescent Health, 63(5), 652-656.

Bednarczyk, Robert A (2019). Abordando os mitos da vacina contra o HPV: informações práticas para profissionais de saúde. Vacinas e imunoterapêuticas humanas, 15 (7-8), 1628-1638.

Burke, N. J., Do, H. H., Talbot, J., Sos, C., Ros, S. \& Taylor, V. M. (2015). Protegendo nossas filhas Khmer: fantasmas do passado, futuro incerto e a vacina contra o papilomavírus humano. Etnia e saúde, 20 (4), 376-390.

Brasil, C. N. S. (2012). Resolução n 466, de 12 de dezembro de 2012. Diário Oficial [da] República Federativa do Brasil.

Dorell, C., Yankey, D., Jeyarajah, J., Stokley, S., Fisher, A., Markowitz, L. \& Smith, P. J. (2014). Atraso e recusa da vacina do papilomavírus humano para meninas, National Immunization Survey - Teen, 2010. Clinical pediatrics, 53 (3), 261-269.

Fernandez-Pineda, M., Cianelli, R., Villegas, N., Matsuda, Y., Parra, E. S. I., \& Montano, N. .P (2020). Fatores salientes entre pais hispânicos em comunidades rurais do sul da Flórida para vacinar seus filhos contra o papilomavírus humano. Jornal de enfermagem pediátrica, 54, $24-33$.

Forman, D., Bray, F., Brewster, D. H., Gombe Mbalawa, C., Kohler, B., Piñeros, M., \& Ferlay, J. (2013). Cancer incidence in five continents, Vol. X. IARC scientific publication, 164.

Forster, A. S.., Rockliffe, L., Marlow, L. A, Bedford, H., McBride, E., \& Waller, J. (2017). Explorando a recusa da vacinação contra o papilomavírus humano entre as minorias étnicas na Inglaterra: Um estudo qualitativo comparativo. Psico - oncologia, 26 (9), 1278-1284.

Gilbert, N. L., Gilmour, H., Dubé, È., Wilson, S. E., \& Laroche, J. (2016) Estimativas e determinantes da não vacinação contra o HPV e recusa da vacina em meninas de 12 a 14 anos de idade no Canadá: resultados da Pesquisa Nacional de Cobertura de Imunização da Infância, 2013. Human vaccines \& immunotherapeutics, 12(6), 1484-1490.

Gilkey, M. B., Calo, W. A., Marciniak, M. W., \& Brewer, N. T. (2017). Pais que recusam ou adiam a vacina contra o HPV: Diferenças no comportamento de vacinação, crenças e preferências de comunicação clínica. Human vaccines \& immunotherapeutics, 13(3), 680-686.

Giraldo, P. C., Silva, M. J. P., Fedrizzi, E. N., Gonçalves, A. K. S., Amaral, R. L. G., Junior, J. E. \& Figueiredo, I. V. (2008). Prevenção da infecção por HPV e lesões associadas. DST-J bras Doenças Sex Transm, 20(2), 132-140.

Godoi, G. R., Melo, L. B., \& Silva, P. S. (2019). A importância da conscientização da família para o melhor alcance da vacina contra o HPV. Centro Universitário Unievangélica.

Grandahl, M., Oscarsson, M., Stenhammar, C., Nevéus, T., Westerling, R. \& Tydén, T. (2014). Não é o momento certo: por que os pais se recusam a permitir que suas filhas recebam a vacinação contra o papilomavírus humano. Acta paediatrica, 103(4), 436-441.

Grandahl, M., Tydén, T., Westerling, R., Nevéus, T., Rosenblad, A., Hedin, E. \& Oscarsson, M. (2017). Para consentir ou recusar a vacinação contra o HPV: um estudo piloto no início do programa nacional de vacinação escolar na Suécia. Journal of school health, 87 (1), $62-70$.

Guedes, M. D. C. R., São Bento, P. A. D. S., Telles, A. C., Queiroz, A. B. A., \& Xavier, R. B. (2017). A vacina do papilomavírus humano e o câncer do colo do útero: uma reflexão. Rev. enferm. UFPE online, 224-231.

Guzman-Holst, A., DeAntonio, R., Prado-Cohrs, D. \& Juliao, P. (2020). Barriers to vaccination in Latin America: A systematic literature review. Vaccine, $38(3), 470-481$

Gyan, K. V., Lechuga, J., Jenerette, C. M., Palmer, M. H., Moore, A. D., \& Hamilton, J. B. (2019). Aceitação da vacina contra o HPV entre mães afroamericanas e suas filhas: uma investigação baseada na cultura. Ethnicity \& health, 24(3), 323-340. 
Research, Society and Development, v. 11, n. 1, e28211124792, 2022

(CC BY 4.0) | ISSN 2525-3409 | DOI: http://dx.doi.org/10.33448/rsd-v11i1.24792

Hirth, J. M., Fuchs, E. L., Chang, M., Fernandez, M. E., \& Berenson, A. B. (2019). Variaç̃̃es no motivo da intenção de não vacinar ao longo do tempo, região e por raça / etnia, NIS-Teen (2008-2016). Vaccine, 37(4), 595-601.

IBGE, Instituto Brasileiro de Geografia e Estatística (2016). Ministério do Planejamento, Orçamento e Gestão. Instituto Brasileiro de Geografia e Estatística. Pesquisa Nacional de Saúde do Escolar 2015.

Kinder, F. D. (2018). Recusa dos pais da vacina contra o papilomavírus humano: estudo em vários locais. Journal of Pediatric Health Care, 32 (2), 150-156.

Krawczyk, A., Perez, S., King, L., Vivion, M., Dubé, E. \& Rosberger, Z. (2015). Tomada de decisão dos pais sobre a vacina do papilomavírus humano para suas filhas: II. Resultados qualitativos. Vacinas e imunoterapêuticas humanas, 11(2), 330-336.

LaMontagne, D. S., Barge, S., Thi Le, N., Mugisha, E., Penny, M. E., Gandhi, S., \& Jumaan, A. O. (2011). Estratégias de entrega da vacina contra o papilomavírus humano que alcançaram alta cobertura em países de baixa e média renda. Boletim da Organização Mundial da Saúde, 89, 821-830.

Lara, LAD S. (2019). A expressão marcante da sexualidade nas adolescentes e no início da vida sexual cada vez mais precocemente. Femina, 205-207.

Lee, H., Kim, M., Kiang, P., Shi, L., Tan, K., Chea, P. \& Grigg-Saito, DC (2016). Fatores associados à vacinação contra o HPV entre adolescentes cambojanos americanos. Public Health Nursing, 33(6), 493-501.

Lipold, L., Sikon, A. \& Rome, E. (2013). Human papillomavirus vaccine: safe, effective, underused. Cleveland Clinic journal of medicine, 80(1), 49.

Lobão, W. M. (2018). Avaliação da aceitação parental da vacina HPV após sua introdução no programa nacional de imunização (Doctoral dissertation, Tese de Doutorado, Fundação Oswaldo Cruz, Salvador, BA, Brasil).

Lopalco, P. L. (2017). Spotlight on the 9-valent HPV vaccine. Drug design, development and therapy, 11, 35.

Luciani, S., Bruni, L., Agurto, I. \& Ruiz-Matus, C. (2019). Implementação e monitoramento da vacina contra HPV na América Latina. salud pública de méxico, 60, 683-692.

McLaughlin-Drubin, M. E., \& Münger, K. (2009). Oncogenic activities of human papillomaviruses. Virus research, 143(2), 195-208.

Melnyk, B. M., \& Fineout-Overholt, E. (2011). Evidence-based practice in nursing e healthcare. A guide to best practice. Philadelphia: Lippincot Williams \& Wilkins.

Ministério da Saúde (2017). Guia Prático sobre o HPV - Perguntas e Respostas. Brasília: Ministério da Saúde. https://antigo.saude.gov.br/images/pdf/2017/dezembro/07/Perguntas-e-respostas-HPV-.pdf.

Ministério da Saúde (2018). Informe técnico da ampliação da oferta das vacinas papilomavírus humano 6, 11, 16 e 18 (recombinante): vacina HPV quadrivalente e meningocócica C (conjugada). Brasília: Secretaria de Vigilância em Saúde, 2018. 39

Mizuta, A. H., Succi, G. D. M., Montalli, V. A. M., \& Succi, R. C. D. M. (2018). Percepções acerca da importância das vacinas e da recusa vacinal numa escola de medicina. Revista Paulista de Pediatria, 37, 34-40.

Moura, L. D. L. (2019). Cobertura vacinal contra o Papilomavírus Humano (HPV) em meninas e adolescentes no Brasil: análise por coortes de nascimentos (Doctoral dissertation).

Oliveira, P. M. N. D., Lignani, L. K., Conceição, D. A. D., Farias, P. M. C. D. M., Takey, P. R. G., Maia, M. D. L. D. S. \& Camacho, L. A. B. (2020). O panorama da vigilância de eventos adversos pós-vacinação ao fim da década de 2010: importância, ferramentas e desafios. Cadernos de Saúde Pública, 36, e00182019.

WHO- World Health Organization (2017). Global Health Sector Strategy on Sexually Transmitted Infections 2016-2021: Implementation Framework for the African Region: Secretariat Report (No. AFR/RC67/7). Regional Office for Africa.

Patel, C., Brotherton, J. M., Pillsbury, A., Jayasinghe, S., Donovan, B., Macartney, K., \& Marshall, H. (2018). O impacto de 10 anos de vacinação contra o papilomavírus humano (HPV) na Austrália: que carga de doença adicional uma vacina não valente prevenirá? Eurosurveillance, 23 (41), 1700737.

Peters, M. D. J., Godfrey C, McInerney P., Munn Z., Tricco A. C, \& Khalil, H. (2020) Capítulo 11: Revisões do escopo (versão 2020). JBI Manual for Evidence Synthesis, JBI. Aromataris.

Podgorski, T., Andrade, V. R. M., Vargas, F. A., \& Oliveira, T. B. (2019). Adesão de adolescentes à vacinação contra o Papilomavírus Humano em um município da Região Sul do Brasil. Revista de Epidemiologia e Controle de Infecção, 9(4), 258-263.

Restivo, V., Costantino, C., Fazio, T. F., Casuccio, N., D’Angelo, C., Vitale, F \& Casuccio, A. (2018). Factors associated with HPV vaccine refusal among young adult women after ten years of vaccine implementation. International journal of environmental research and public health, 15(4), 770.

Sanchez, V., Tovar-Aguirre, O. L., Franco, S., Ortiz, N. E. A., Louie, K., Sanchez, G. I., \& Garces-Palacio, I. C. (2019). Percepção sobre as barreiras e facilitadores do programa de vacina contra o HPV nas escolas de Manizales, Colômbia: um estudo qualitativo em meninas matriculadas na escola e seus pais. Preventive medicine reports, 16, 100977.

Santos, G. B. G. D. (2018). Comportamento de risco à saúde em relação à prevenção de câncer de boca e orofaringe de adolescentes estudantes de uma Instituição Federal de Ensino de Alagoas.

Santos, J. G. S. \& Dias, J. M. G. (2018). Vacinação pública contra o papilomavirus humano no Brasil. Rev Med Minas Gerais, 28(1), 1-7. 
Research, Society and Development, v. 11, n. 1, e28211124792, 2022

(CC BY 4.0) | ISSN 2525-3409 | DOI: http://dx.doi.org/10.33448/rsd-v11i1.24792

SBIM - Sociedade Brasileira de Imunizações (2020). Coberturas vacinais no Brasil são baixas e heterogêneas, mostram informações do PNI. https://sbim.org.br/noticias/1359-coberturas-vacinais-no-brasil-sao-baixas-e-heterogeneas-mostram-informacoes-do-pni.

Schwarz, T. F., Huang, L. M., Valencia, A., Panzer, F, Chiu, C. H., Decreux, A., \& Struyf, F. (2019). A ten-year study of immunogenicity and safety of the AS04-HPV-16/18 vaccine in adolescent girls aged 10-14 years. Human vaccines \& immunotherapeutics, 15(7-8), 1970-1979.

Silva, M. R. B., Conceição, A. S. F., Silva, D. M., Araujo Ramado, A. D., Andrade, J. G., Reis, T. L., \& Souza, R. S. (2020). Eventos adversos pós-vacinais papilomavírus humano em adolescentes. Saúde Coletiva (Barueri), 10(53), 2470-2489.

Silva, P. M. C. D., Silva, I. M. B., Interaminense, I. N. D. C. S., Linhares, F. M. P., Serrano, S. Q., \& Pontes, C. M. (2018). Conhecimento e atitudes sobre o Papilomavírus humano e a vacinação. Escola Anna Nery, 22.

Siu, J. Y. M. (2014). Percepções e barreiras à vacinação de filhas contra o Papilomavírus Humano (HPV) entre mães em Hong Kong. BMC women's health, 14(1), 1-10

Souza, W. M., da Silva, A. D., Santos, A. D., Soares, S., Morone, A. R. \& Santos, F. (2020). Análise da imunização contra o HPV no Brasil: um estudo ecológico exploratório de 2016 a 2018-Hpv immunization analysis in brazil: an exploratory ecological study from 2016 to 2018 . Revista Eletrônica de Ciência, Tecnologia e Inovação em Saúde, 1, 1-9.

Szilagyi, P. G., Albertin, C. S., Gurfinkel, D., Saville, A. W, Vangala, S., Rice, J. D., \& Kempe, A. (2020). Prevalência e características da hesitação à vacina contra o HPV entre pais de adolescentes nos Estados Unidos. Vaccine, 38 (38), 6027-6037.

Tatar, O., Shapiro, G. K., Perez, S., Wade, K. \& Rosberger, Z. (2019). Usando o modelo de processo de adoção por precaução para esclarecer a hesitação da vacina contra o papilomavírus humano em pais canadenses de meninas e pais de meninos. Vacinas humanas e imunoterapêuticos.

Thomas, T. L., Caldera, M., \& Maurer, J. (2019). Um breve relatório: o conhecimento dos pais sobre a vacina contra o HPV na zona rural do sul da Flórida. Vacinas humanas e imunoterapêuticos.

Tricco, A. C., Lillie, E., Zarin, W., O'Brien, K. K., Colquhoun, H., Levac, D., \& Straus, S. E. (2018). Extensão PRISMA para análises de escopo (PRISMAScR): lista de verificação e explicação. Annals of internal medicine, 169 (7), 467-473.17. Ouzzani M., Hammady H, Fedorowicz Z, Elmagarmid Ahmed. Rayyan - a web and mobile app for systematic reviews. Systematic Reviews. 2016.

Villa, A, Patton, L. L., Giuliano, A. R., Estrich, C. G., Pahlke, S. C., O'Brien, K. K., \& Araujo, M. W. (2020). Resumo das evidências sobre a segurança, eficácia e eficácia das vacinas do papilomavírus humano: Revisão geral de revisões sistemáticas. The Journal of the American Dental Association, 151 (4), 245-254.

Waller, J., Forster, A., Ryan, M., Richards, R., Bedford, H. \& Marlow, L. (2020). Tomada de decisão sobre a vacinação contra o HPV em pais de meninos e meninas: uma pesquisa de base populacional na Inglaterra e no País de Gales. Vaccine, 38(5), 1040-1047.

Ward, JK, Crépin, L., Bauquier, C., Vergelys, C., Bocquier, A., Verger, P. \& Peretti-Watel, P. (2017). 'Não sei se estou tomando a decisão certa': mães francesas e a vacinação contra o HPV em um contexto de polêmica. Health, Risk \& Society, 19 (1-2), 38-57.

World Health Organization. Information centre on HPV and cervical cancer (HPV Information Centre): summary report on HPV and cervical cancer statistics in South Africa [Internet]. World Health Organization, c2013 